\title{
COMPARACIÓN HISTOLÓGICA DEL ESPESOR DE LA CAPA MEDIA DE LAS ARTERIOLAS PULMONARES EN POLLOS DE CARNE COBB-VANTRESS Y ROSS EXPUESTOS A HIPOXIA
}

\author{
Histological Comparison of Layer Media Thickness of Pulmonary \\ Arterioles between Cobb-Vantress and Ross Broiler Chicken Exposed \\ TO HYPOXIA
}

\author{
José Rodríguez G. ${ }^{1,5}$, María Vásquez C. ${ }^{1}$, Sergio Cueva M. ${ }^{1}$, Milder Ayón S. ${ }^{1}$, Boris \\ Lira M. ${ }^{1}$, Alfonso Chavera C. ${ }^{2}$, Pedro Angulo H. ${ }^{3}$, Néstor Falcón P. ${ }^{4}$
}

\section{RESUMEN}

El presente estudio tuvo por objetivo determinar el desarrollo muscular de las arteriolas pulmonares mediante la relación de la capa muscular lisa arteriolar pulmonar/diámetro arteriolar(CM/DA). Se utilizaron 90 pollos de la línea Cobb-Vantress y 90 de la línea Ross. De estos, 10 pollos de cada línea fueron pesados y sacrificados al primer día de edad, y el resto fue distribuido al azar en un grupo criado a nivel de mar $(\mathrm{n}=40)$ y otro a $3320 \mathrm{msnm}$ $(\mathrm{n}=40)$, donde fueron trasladados al primer día de edad. Diez aves de cada subgrupo fueron pesadas y sacrificadas a los 10,20,30 y 40 días de edad. Se determinó la relación $\mathrm{CM} / \mathrm{DA}$ en cortes histológicos de pulmón coloreados con hematoxilina fosfotúngstica de Mallory. Las aves criadas a nivel del mar ganaron más peso que las criadas en altura $(\mathrm{p}<0.05)$ y las aves de la línea Ross fueron las que obtuvieron un mayor peso a nivel del mar pero menor peso en altura que las aves de la línea Cobb-Vantress $(p<0.05)$. La relación $\mathrm{CM} / \mathrm{DA}$ en todos los grupos aumentó con la edad $(\mathrm{p}<0.05)$, pero la línea CobbVantres a nivel del mar presentó la mayor relación CM/DA a partir de los 20 días de edad $(\mathrm{p}<0.05)$.

Palabras clave: histología, músculo liso, arteriola pulmonar, hipoxia, pollos

\section{Abstract}

The objective of the present study was to determine the development of the smooth muscle of pulmonary arterioles through the relationship of the smooth muscle layer of pulmonary arteriole/arteriolar diameter (CM/DA). Ninety Cobb-Vantress chicks and 90 Ross chicks were used. Of these, 10 birds were weighed and slaughtered at 1 day of age,

1 Laboratorio de Fisiología Animal, ${ }^{2}$ Laboratorio de Histología, Embriología y Patología Veterinaria, ${ }^{3}$ Laboratorio de Farmacología y Toxicología Veterinaria, Facultad de Medicina Veterinaria, Universidad Nacional Mayor de San Marcos, Lima

${ }^{4}$ Facultad de Veterinaria y Zootecnia, Universidad Peruana Cayetano Heredia, Lima

5 E-mail:joserodriguezmv@gmail.com 
and the others were randomly distributed in a group raised at sea level $(n=40)$ and a group raised at $3320 \mathrm{~m}$ above the sea level $(n=40)$. Ten birds per group were weighed and slaughtered at 10, 20,30 and 40 days of age. The CM/DA relation was calculated in lung slides stained with Mallory's phosphotungstic acid hematoxylin. Birds raised at sea level weighed more than those raised at high altitude $(p<0.05)$, and chicks of the Ross line obtained higher body weight at sea level but lower at high altitude than those of the Cobb-Vantress line $(\mathrm{p}<0.05)$. The $\mathrm{CM} / \mathrm{DA}$ relationship increased in all groups according to age $(\mathrm{p}<0.05)$, but the Cobb-Vantress line at sea level showed the higher CM/DA relationship from 20 days of age onwards $(\mathrm{p}<0.05)$.

Key words: histology, smooth muscle, pulmonary arteriole, hypoxia, chicks

\section{INTRODUCCIÓN}

La frecuencia de presentación del síndrome ascítico en pollos de carne a nivel de mar se ha incrementado en los últimos años, debido a la selección genética del pollo de engorde, basada en un incremento de su velocidad de crecimiento y depósito de masa muscular. Este criterio de selección trae como consecuencia un aumento en la carga metabólica debido a la mayor demanda de oxígeno, haciendo al pollo más susceptible de presentar hipertensión arterial pulmonar, ante una mayor vasoconstricción y desarrollo muscular liso de las arteriolas pulmonares (Cortés et al., 2006; Francia et al., 2009).

La mejor conversión de alimento en carne que presentan las líneas genéticas actuales (Ross y Cobb-Vantress) a comparación de sus antecesoras, viene resultando en una desarrollo inadecuado del sistema cardiorespiratorio acorde con su crecimiento y, por tanto, con sus exigencias metabólicas, favoreciendo el desarrollo de cuadros de ascitis (Druyan et al., 2008). Este cuadro se agrava si converge con la presencia de altos niveles de gases, como el amoniaco, así como con una deficiente ventilación en los galpones, convirtiendo al ambiente del galpón en un lugar con bajos niveles de oxígeno (Al-Homidan et al., 2003). Estos factores, sumado a la alta exigencia metabólica de los pollos de carne lleva al animal a una hipoxia tisular, desencadenando la producción de sustancias vasoconstrictoras como catecolaminas, angiotensina II, endotelina-1 y otros, que a su vez tienen efectos mitogénicos a nivel de la capa muscular lisa vascular de las arteriolas pulmonares (Bendall et al., 2002; Hassoun et al., 2004).

La vasoconstricción pulmonar es el mecanismo primario del sistema vascular pulmonar que se presenta ante la hipoxia, siendo las arteriolas pulmonares distales o de resistencia, el principal sitio anatómico de respuesta (Sylvester et al., 2001). En las especies animales donde la hipoxia aguda provoca una vasoconstricción marcada, la hipoxia crónica provoca una hipertensión pulmonar sostenida, a causa de un aumento en la cantidad de fibra muscular lisa de la capa media de las arteriolas pulmonares (Cunningham, 2009); es decir, a mayor grado de proliferación o hipertrofia de la capa muscular con respecto al diámetro de la arteriola pulmonar es mayor la posibilidad de presentar hipertensión arterial pulmonar (HAP) (Meyrick y Reid, 1978; Rabinovitch et al., 1979; Jeffery y Wanstall, 2001) y, por consiguiente, la posibilidad de desarrollar síndrome ascítico.

El presente estudio se planteó con el objetivo de evaluar el grosor de la capa muscular lisa de las arteriolas pulmonares (CM/ 
DA) durante la crianza en pollos de dos líneas de carne a nivel de mar y en altura.

\section{Materiales y Métodos}

\section{Lugar de Ejecución}

El estudio se realizó en el Laboratorio de Fisiología Animal de la Facultad de Medicina Veterinaria de la Universidad Nacional Mayor de San Marcos, localizada en la ciudad de Lima, a 150 metros de altitud, presión barométrica de $750 \mathrm{mmHg}$ y una presión parcial de oxígeno de $157 \mathrm{mmHg}$, y en la estación experimental del Instituto Veterinario de Investigaciones Tropicales y de Altura (IVITA) - El Mantaro, provincia de Jauja, departamento de Junín, a una altitud de 3320 msnm, presión barométrica de $510 \mathrm{mmHg}$ y presión parcial de oxígeno de $107 \mathrm{mmHg}$.

\section{Animales y Muestras}

Se utilizaron 180 aves de dos líneas comerciales de pollos de carne: Cobb-Vantress (90 aves) y Ross (90 aves). De estos, 10 pollos de cada línea fueron pesados y sacrificados al primer día de edad, y el resto fue distribuido al azar en dos grupos: uno criado a nivel de mar $(\mathrm{n}=40)$ y otro a $3320 \mathrm{msnm}$ $(\mathrm{n}=40)$, donde fueron trasladados al primer día de edad. Las aves fueron criadas siguiendo los estándares de crianza para producción de pollos comerciales de carne.

Se sacrificaron 10 animales por grupo a los 10, 20, 30 y 40 días de edad. El sacrificio fue por desarticulación occípito-atloidea. Se removieron los pulmones y fueron colocados en frascos con solución fijadora (formol tamponado al 10\%), dejándose en reposo por 72 horas (AFIP, 1992).

Las aves fueron pesadas antes del sacrificio, utilizando una balanza digital en gramos.

\section{Procesamiento de Muestras}

La parte media de los pulmones fueron reducidas a un tamaño aproximado de $0.5 \mathrm{x}$ $0.5 \times 0.5 \mathrm{~cm}$. Las muestras fueron incluidas en parafina, se hicieron cortes de $5 \mu \mathrm{m}$ de espesor y se colocaron en láminas portaobjetos (AFIP, 1992). Las láminas fueron coloreadas con hematoxilina fosfotúngstica de Mallory, que da una coloración azul claro para las fibras musculares y un color naranja rojizo para las fibras colágenas (Montalvo et al., 1979).

Las secciones del parénquima pulmonar se observaron en un microscopio binocular Carl Zeiss, con oculares WF 10x/20x y objetivos de 4x, 10x, 40x y 100x. La observación de las imágenes se realizó a 400x. Para la medición en micras de las variables se incluyó una regla micrométrica en uno de los oculares. Se midieron los diámetros arteriolares (DA) externos mediante dos mediciones perpendiculares entre sí, desde la parte externa de la capa adventicia, considerándose el promedio de las dos mediciones como el diámetro de la arteriola. El grosor de la capa muscular (CM) se midió en dos puntos y se obtuvo el promedio (Fig. 1).

En cada sección de parénquima pulmonar se midieron 10 arteriolas con diámetros externos entre 20 y $150 \mu \mathrm{m}$. Con estas mediciones se calculó la relación grosor de capa muscular lisa arteriolar pulmonar/diámetro arteriolar (CM/DA) (Montalvo et al., 1979; Heath et al., 1981).

\section{Análisis Estadístico}

El peso y la relación CM/DA entre grupos de pollos de una misma edad se evaluaron mediante la prueba de " $t$ " Student con un nivel de confianza del 95\%. La comparación entre edades se hizo con la prueba de Tuckey con un nivel de confianza del $95 \%$ y poder de prueba de $80 \%$. Los resultados de los grupos se expresan como promedio y desvío estándar. 


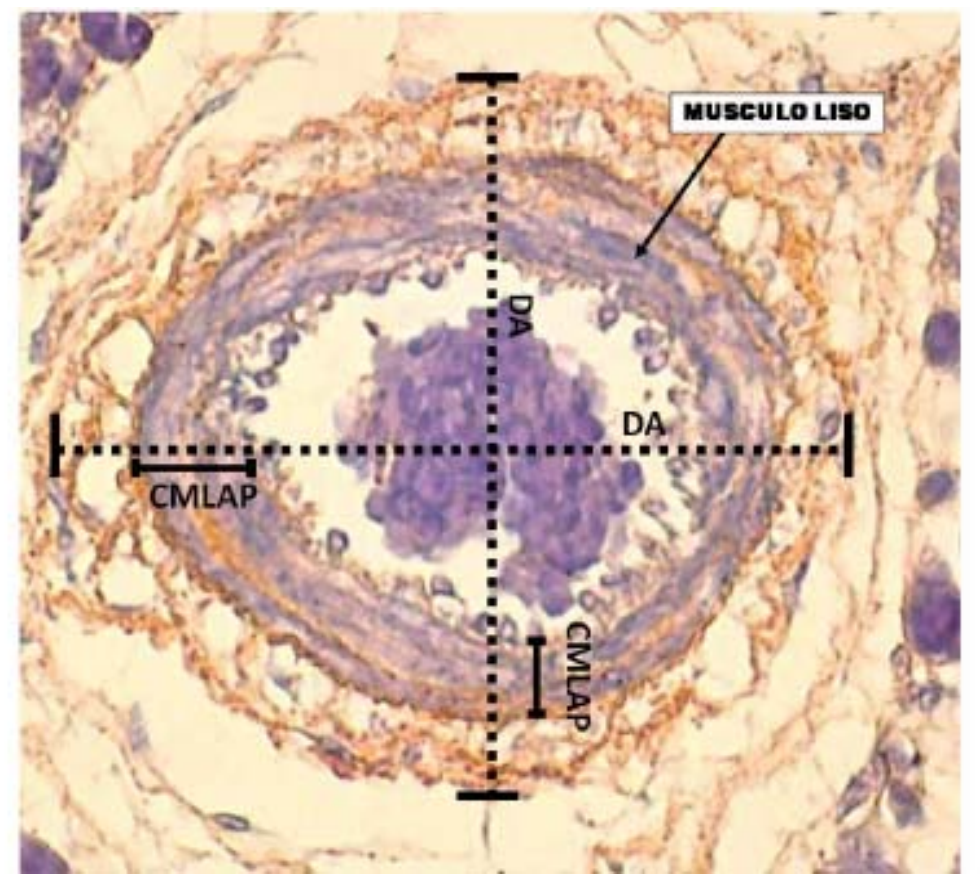

Figura 1. Corte de pulmón de pollo de 20 días de edad. CMLAP (Capa media lisa de arteriolas pulmonar), DA (diámetro arteriolar). Coloración: Hematoxilina fosfotúngstica de Mallory

\section{RESUltados}

Las aves criadas a nivel del mar ganaron más peso que las criadas en altura $(\mathrm{p}<0.05)$. Estas diferencias se hacen evidentes a partir de los 10 días de edad. Asimismo, las aves de la línea Ross fueron las que obtuvieron un mayor peso a nivel del mar pero el menor peso en altura $(\mathrm{p}<0.05)$ (Cuadro 1$)$.

La relación $\mathrm{CM} / \mathrm{DA}$ fue similar a los 20 días de edad, independientemente de la línea genética y la zona de crianza, pero a edades mayores, esta relación fue mayor en las aves de la línea Cobb-Vantres en las dos zonas de crianza $(\mathrm{p}<0.05)$, con excepción del grupo Ross a los 40 días de edad (Cuadro 2).

Al comparar la relación $\mathrm{CM} / \mathrm{DA}$ por edades y dentro de cada línea y zona de crianza se observó un incremento de la relación hasta los 30 días $(p<0.05)$, e incluso hasta los 40 días de edad en las aves de la línea Ross criados en altura (Cuadro 2).

\section{Discusión}

En el presente estudio, se pudo observar en crianza a nivel del mar de la línea CobbVantress, especialmente a los 30 días de edad, que es la etapa de mayor crecimiento acelerado, un mayor grosor de la capa muscular lisa de las arteriolas pulmonares (Cuadro 2) y un menor peso corporal (Cuadro 1) que la línea Ross, lo cual indica una menor resistencia a una exigencia metabólica mayor; por otro lado, la línea Ross estaría demostrando una mejor adaptación a esta alta tasa metabólica a nivel del mar.

Las arteriolas pulmonares reaccionan a la hipoxia presentando vasoconstricción, causando un aumento de la presión intravascular e hipertrofia de la pared muscular, lo que a su vez aumenta la resistencia vascular incrementando la presión arterial pulmonar (Pries et al., 2005; Moreno de Sandino y Hernández, 2006). En la altura, ambas líneas mostraron una disminución marcada en la 
Cuadro 1. Peso (g) de pollos de carne de las líneas Cobb-Vantress y Ross criados a nivel del mar $y$ en altura ( $3320 \mathrm{msnm})$

\begin{tabular}{cccccccccc}
\hline & \multicolumn{3}{c}{ Cobb-Vantress } \\
\cline { 2 - 9 } $\begin{array}{c}\text { Edad } \\
\text { días })\end{array}$ & \multicolumn{2}{c}{ Nivel del mar } & \multicolumn{2}{c}{ Altura } & \multicolumn{2}{c}{ Nivel del mar } & \multicolumn{2}{c}{ Altura } \\
\cline { 2 - 10 } & Promedio & d.e. & Promedio & d.e. & Promedio & d.e. & Promedio & d.e. \\
\hline 1 & $56.2^{\mathrm{a}}$ & 2.9 & $56.2^{\mathrm{a}}$ & 2.9 & $45.6^{\mathrm{b}}$ & 3.2 & $45.6^{\mathrm{b}}$ & 3.2 \\
10 & $186.3^{\mathrm{a}}$ & 20.7 & $111.7^{\mathrm{c}}$ & 9.4 & $237.4^{\mathrm{b}}$ & 15.2 & $113.3^{\mathrm{c}}$ & 11.1 \\
20 & $689.7^{\mathrm{a}}$ & 71.1 & $406.0^{\mathrm{c}}$ & 32.3 & $755.4^{\mathrm{b}}$ & 50.9 & $397.3^{\mathrm{c}}$ & 50.8 \\
30 & $1503.3^{\mathrm{a}}$ & 179.9 & $934.3^{\mathrm{d}}$ & 114.2 & $1645.7^{\mathrm{b}}$ & 125.5 & $748.1^{\mathrm{c}}$ & 134.5 \\
40 & $2570.2^{\mathrm{a}}$ & 76.1 & $1709.1^{\mathrm{d}}$ & 228.9 & $2778.0^{\mathrm{b}}$ & 90.2 & $1356.0^{\mathrm{c}}$ & 86.6 \\
\hline a,b,c,d & Letras diferentes indican diferencia estadistica significativa $(\mathrm{p}<0.05)$ entre nivel del mar y altura \\
\multicolumn{3}{c}{ a una misma edad } & & & & & &
\end{tabular}

Cuadro 2. Relación CM/DA (grosor de la capa muscular / diámetro arteriolar) de pollos de carne de las líneas Cobb-Vantress y Ross criados a nivel del mar y altura (3320 msnm)

\begin{tabular}{cccccccccc}
\hline \multirow{3}{*}{$\begin{array}{c}\text { Edad } \\
\text { (días) }\end{array}$} & \multicolumn{4}{c}{ Cobb-Vantress } & \multicolumn{5}{c}{ Noss } \\
\cline { 2 - 9 } & Promel del mar & \multicolumn{2}{c}{ Altura } & \multicolumn{3}{c}{ Nivel del mar } & \multicolumn{2}{c}{ Altura } \\
\hline 1 & $0.104^{\mathrm{a} 1}$ & 0.028 & $0.104^{\mathrm{a} 1}$ & 0.028 & $0.105^{\mathrm{a} 1}$ & 0.022 & $0.105^{\mathrm{a} 1}$ & 0.022 \\
10 & $0.114^{\mathrm{a} 2}$ & 0.019 & $0.117^{\mathrm{a} 2}$ & 0.021 & $0.113^{\mathrm{a} 2}$ & 0.034 & $0.112^{\mathrm{a} 2}$ & 0.015 \\
20 & $0.130^{\mathrm{a} 3}$ & 0.019 & $0.128^{\mathrm{a} 3}$ & 0.033 & $0.122^{\mathrm{b} 3}$ & 0.015 & $0.121^{\mathrm{b} 3}$ & 0.012 \\
30 & $0.142^{\mathrm{a} 4}$ & 0.025 & $0.139^{\mathrm{a} 4}$ & 0.017 & $0.132^{\mathrm{b} 4}$ & 0.018 & $0.135^{\mathrm{b} 4}$ & 0.015 \\
40 & $0.148^{\mathrm{a} 4}$ & 0.024 & $0.144^{\mathrm{a} 4}$ & 0.025 & $0.138^{\mathrm{b} 4}$ & 0.019 & $0.142^{\mathrm{ab} 5}$ & 0.015 \\
\hline
\end{tabular}

${ }^{a, b}$ Letras diferentes indican diferencia estadística significativa $(p<0.05)$ entre nivel del mar y altura a una misma edad

1,2,3,4,5 Números diferentes indican diferencia estadística significativa $(p<0.05)$ entre edades dentro de un mismo grupo de crianza

ganancia de peso, pero sin llegar a observarse diferencias significativas en la relación $\mathrm{CM} / \mathrm{DA}$ con sus homólogos en crianza a nivel del mar (Cuadro 2). Estudios realizados por Cortés et al. (2006) demuestran que en los últimos años, la selección genética del pollo de engorde ha permitido un incremento en la velocidad de crecimiento y depósito de masa 
muscular, que trae como consecuencia un aumento en la carga metabólica ante una mayor demanda de oxígeno. Esta mayor demanda de oxígeno en el broiler causa una hipoxemia crónica que induce a una vasoconstricción con proliferación del musculo liso arteriolar pulmonar, haciendo que estos animales sean más susceptibles a presentar cuadros de hipertensión arterial pulmonar e hipertrofia cardiaca derecha (Jeffery y Wanstall, 2001), pues existe una relación directa entre la hipoxemia, aumento del grosor de arteriolas pulmonares, hipertrofia cardiaca e hipertensión arterial pulmonar.

Asimismo, en un estudio con varias líneas de pollos, se encontró que la línea Ross presenta una mayor ganancia de peso corporal con un menor desarrollo del ventrículo derecho en comparación con la línea CobbVantress (Gonzáles et al., 1998), lo cual se asemeja a los resultados del presente estudio, si se considera la directa relación entre muscularización arteriolar pulmonary aumento del tamaño del ventrículo derecho (Staub, 1963; Olkowski et al., 2005). Por otro lado, Arce (1991) y Berger (1992) determinaron que la hipertensión pulmonar está muy relacionada a la vasoconstricción producto de la muscularización de las arteriolas pulmonares.

La mayor presentación de cuadros de hipertensión arterial y síndrome ascítico en pollos broilers se da entre la tercera y quinta semana de edad (Hernández, 1986; Francia et al., 2009), lo cual concuerda con el presente estudio, donde a partir de los 10 días de edad se incrementa la relación CM/DA en todos los grupos y a partir del día 20 de edad se encuentran diferencias entre líneas. Esto estaría indicando que los procesos de remodelación de las arteriolas pulmonares estarían influyendo en la presentación de los cuadros de hipertensión arterial pulmonar y síndrome ascítico. Esta etapa de la vida del ave es considerada como la etapa de crecimiento acelerado (Mack y Donald, 1998).

Ambas líneas de pollos criadas en la altura tuvieron menor ganancia de peso que sus pares a nivel del mar $(\mathrm{p}<0.05)$, lo cual indicaría que la baja presión parcial de oxígeno ambiental fuerza al animal a disminuir su requerimiento de oxígeno a nivel tisular, lo que se logra con la disminución de metabolismo, y por lo tanto, disminuyendo la ganancia de peso corporal. De no ser así, ocurriría un mayor engrosamiento de la musculatura arteriolar que devendría en la presentación de cuadros de hipertensión arterial pulmonar (Arce et al., 1988).

\section{ConClusiones}

- La línea Cobb-Vantres a nivel del mar presenta mayor relación CM/DA (grosor de la capa muscular / diámetro arteriolar) a partir de los 20 días de edad $(\mathrm{p}<0.05)$.

- La relación CM/DA, dentro de cada línea y zona de crianza, mostró un incremento significativo hasta los 30 días $(\mathrm{p}<0.05)$.

- La línea Ross obtuvo el mayor peso corporal a nivel del mar, en tanto que la Línea Cobb-Vantress tuvo el mayor peso corporal a $3320 \mathrm{msnm}(\mathrm{p}<0.05)$

\section{LITERATURA CITADA}

1. [AFIP] Instituto de Patología de las Fuerzas Armadas de los Estados Unidos de América. 1992. Métodos histotecnológicos. Washington DC: AFIP. $279 \mathrm{p}$.

2. Al-Homidan A, Robertson JF, Petchey AM. 2003. Review of the effect of ammonia and dust concentrations on broiler performance. World Poultry Sci J 59: 340-349.

3. Arce J, Magaña C, López C, Vásquez P, Ávila G. 1988. Constantes fisiológicas y parámetros productivos de tres líneas comerciales de pollo de engorde y su relación con el síndrome ascítico. En: Memorias XIII Convención Nacional de la Asociación Nacional de Especialistas en Ciencias Avícolas. Acapulco, México. 
4. Arce J. 1991. Restricción alimenticia para disminuir la ascitis. Avicultura Profesional 8(3): 96-102.

5. Berger M. 1992. La restricción alimenticia en el síndrome ascítico en pollos de engorde. Avicultura Profesional 9(3): 12-22.

6. Bendall J, Cave C, Heymes C, Gall N, Shah A. 2002. Pivotal role of gp91phox-containing NADPH oxidase in angiotensin II-induced cardiac hypertrophy in mice. Circulation 105: 293-296.

7. Cortés A, Estrada A, Ávila E. 2006. Productividad y mortalidad por síndrome ascítico en pollos de engorda alimentados con dietas granuladas y en harina. Téc Pec, Méx 44: 241-246.

8. Cunningham J. 2009. Fisiología respiratoria. $4^{\circ}$ ed. En: Fisiología veterinaria. Barcelona: Elsevier. p 566-617.

9. Druyan S, Hadad Y, Cahaner A. 2008. Tasa de crecimiento en pollos broilers ascitis-resistente versus ascitis-sensible en líneas comerciales y experimentales. Poultry Sci 87: 964-967.

10. Francia M, Icochea $E$, Reyna $P$, Figueroa E. 2009. Registro de mortalidad, eliminados y descartes a la saca de dos líneas genéticas de pollos de carne. Rev Inv Vet, Perú 20: 228-234.

11. Gonzáles E, Buyse J, Sayuri T, Sartori J, Decuypere E. 1998. Metabolic disturbances in male broilers of different strains. Performance, mortality, and right ventricular hypertrophy. Poultry Sci 77: 1646-1653.

12. Heath D, Smith P, Ríos Dalenz J, Williams D, Harris P. 1981. Small pulmonary arteries in some natives of $\mathrm{La}$ Paz, Bolivia. Thorax 36: 599-604.

13. Hernández V. 1986. La ascitis hipóxica en pollos: Influencia y posibles soluciones. En: Memorias VI Seminario Internacional de Patología Aviar. p 95.

14. Hassoun PM, Filippov G, Fogel M, Donaldson C, Kayyali US, Shimoda LA, Bloch KD. 2004. Hypoxia decreases of soluble guanylate cyclase in cultured rat pulmonary artery smooth muscle cells. Am J Resp Cell Mol 30: 908-913.

15. Jeffery TK, Wanstall JC. 2001. Pulmonary vascular remodeling: a target for therapeutic intervention in pulmonary hypertension. Pharmacol Therapeut 92: 1-20.

16. Mack O, Donald D. 1998. Manual de producción avícola. $4^{\mathrm{a}}$ ed. México DF: Manual Moderno. 829 p.

17. Meyrick B, Reid L. 1978. The effect of continued hypoxia on rat pulmonary arterial circulation: an ultrastructural study. Lab Invest 38: 188-200.

18. Montalvo C, Ayón M, Sillau A. 1979. Histología de las arteriolas pulmonares en aves a nivel del mar y en la altura (3300 m). En: Resúmenes VI Congreso Latinoamericano de Avicultura. Lima.

19. Moreno de Sandino M, Hernández H. 2006. Pulmonary arteriole remodeling in hypoxic broilers expressing different amounts of endothelial nitric oxide synthase. Poultry Sci 85: 899-901.

20. Olkowski A, Abbott J, Classen H. 2005. Pathogenesis of ascites in broilers raised at low altitude: aetiological considerations based on echocardiographic findings. J Vet Med Assoc 52: 166-171.

21. Pries A, Reglin B, Secomb T. 2005. Remodeling of blood vessels: Responses of diameter and wall thickness to hemodynamic and metabolic stimuli. Hypertension 46: 652-663.

22. Rabinovitch M, Gamble W, Nadas A, Miettinen O, Reid L. 1979. Rat pulmonary circulation after chronic hypoxia: haemodynamic and structural features. Am J Physiol 236: H818-H827.

23. Staub N. 1963. Site of action of hypoxia on the pulmonary vasculature. Fed Proc 22: 453 [Abstract].

24. Sylvester J, Sham J, Shimoda L, Liu Q. 2001. Cellular mechanisms of acute hypoxic pulmonary vasoconstriction. In: Respiratory-circulatory interactions in health and disease. New York: Informa Healthcare. p 351-359. 